\title{
An unusual cause of panhypopituitarism
}

\author{
RA Higgoda, K Lokuketagoda, D Perera, K Thirumavalavan
}

Ceylon Medical Journal 2017; 62: 246-47

DOI:http://doi.org/10.4038/cmj.v62i4.8578

\section{Introduction}

Intracranial vascular aneurysms are an exceedingly rare cause of hypopituitarism and it only contributes to $0.17 \%$ of all cases of hypopituitarism [1]. We report a patient who was diagnosed with panhypopituitarism secondary to a giant internal carotid artery aneurysm.

\section{Case Presentation}

A 55 year old unmarried male presented with faintishness and postural giddiness of two weeks duration along with long standing impotence and loss of libido. On examination he had an alabaster complexion with absent facial, axillary and pubic hair and testicular atrophy.

There was a significant postural drop in blood pressure. Rest of the physical examination was normal. Laboratory investigations revealed low serum sodium $(128 \mathrm{mmol} / \mathrm{l})$, with normal serum potassium and creatinine levels and normal liver profile. Endocrine profile revealed low random cortisol (with positive short synacthen test) ACTH, TSH, free thyroxine, FSH, LH, testosterone, IGF1 with a normal prolactin level.

Magnetic resonance imaging (MRI) with magnetic resonance angiography (MRA) showed a large fusiform aneurysm of distal petrous and cavernous portions of left internal carotid artery compressing on the pituitary (Figure 1) which was confirmed with digital subtraction angiography (DSA) (Figure 2).

He improved symptomatically with hormone replacement therapy (thyroxine, hydrocortisone and testosterone) and the aneurysm was managed conservatively.

\section{Discussion}

An intracranial aneurysm is an extremely rare cause of hypopituitarism and causes hypopituitarism either by direct compression or vascular compromise of the

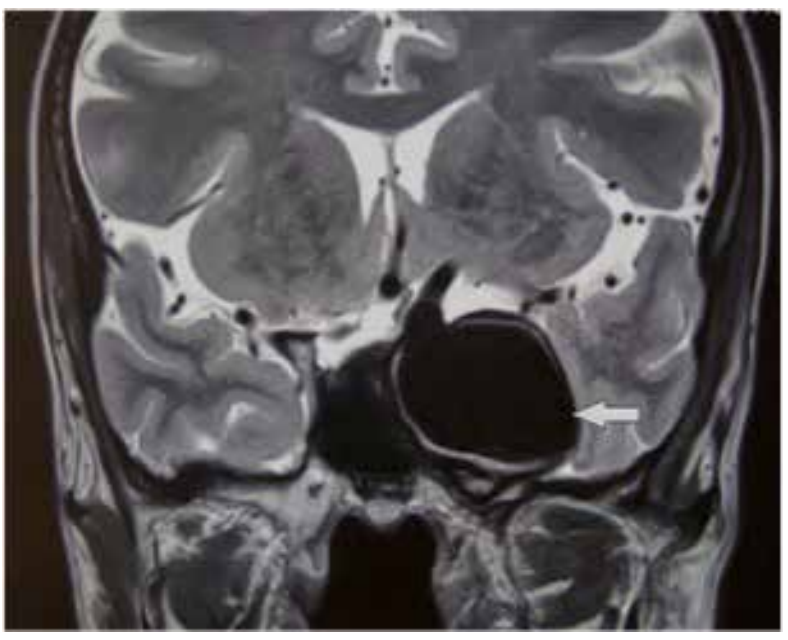

Figure 1. MRI Brain showing a large aneurysm of left internal carotid artery mimicking a space occupying lesion (arrow)

pituitary or as in our case by both mechanisms [1,2,3]. In certain occasions, these aneurysms can even mimic a pituitary adenoma, thus it is crucial to differentiate between a pituitary adenoma and an intracranial aneurysm pre-operatively to avoid potential surgical complications [3]. MRA and DSA play a major role in proper delineation of the vascular anatomy. Preferred modality of treatment of such giant aneurysms is with endovascular flow diverter stents which are not yet available in Sri Lanka [2].

\section{Acknowledgements}

We would like to acknowledge support given by Departments of Interventional Radiology, Chemical Pathology and Endocrinology in investigating this patient.

\section{Conflicts of interest}

Authors declare that there are no conflicts of interest. 


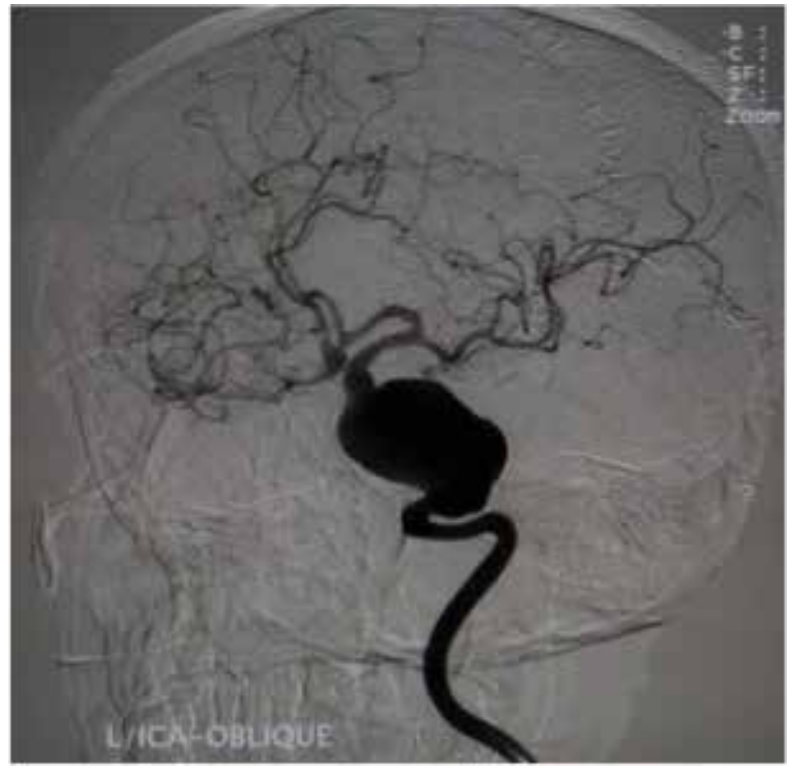

Figure 2. DSA showing a large fusiform aneurysm of distal petrous and cavernous portions of left internal carotid artery

\section{References}

1. Heshmati HM, Fatourechi V, Dagam SA, Piepgras DG. Hypopituitarism Caused by Intrasellar Aneurysms. Mayo Clinic Proceedings 2001;76(8): 789-93.

2. Ding D, Mehta GU, Liu KC. Pituitary insufficiency from large unruptured supraclinoid internal carotid artery aneurysm. $B r J$ Neurosurg 2014;28(2): 290-2.

3. Ooi TC, Russell NA. Hypopituitarism resulting from an intrasellar carotid aneurysm. Can J Neurol Sci 1986;13(1):70-1. 